\title{
GROUP-TWISTED ALEXANDER-WHITNEY AND EILENBERG-ZILBER MAPS
}

\author{
A. V. SHEPLER AND S. WITHERSPOON
}

\begin{abstract}
We define group-twisted Alexander-Whitney and Eilenberg-Zilber maps for converting between bimodule resolutions of skew group algebras. These algebras are the natural semidirect products recording actions of finite groups by automorphisms. The group-twisted chain maps allow us to transfer information between resolutions for use in homology theories, for example, in those governing deformation theory. We show how to translate in particular from the default (but often cumbersome) reduced bar resolution to a more convenient twisted product resolution. This provides a more universal approach to some known results classifying PBW deformations.
\end{abstract}

\section{INTRODUCTION}

Skew group algebras encode actions of groups on other algebras by automorphisms. Deformations of skew group algebras include, for example, symplectic reflection algebras, rational Cherednik algebras, graded Hecke algebras, and Drinfeld orbifold algebras; e.g., see [7, 8, 15, 18]. These are all PBW deformations of $S(V) \rtimes G$ for the action of a finite group $G$ on a finite dimensional vector space $V$ with symmetric algebra $S(V)$. The Hochschild cohomology of $S(V) \rtimes G$ in turn captures important information about its deformations. In positive characteristic, the situation is more complicated than over fields of characteristic 0 , as the group algebra $k G$ itself is not always semisimple. There are a number of recent papers on the representation theory of such deformations in positive characteristic; see, e.g., [1, 2, 3, 6, 17]. In this short note, we show how to understand some prior results on PBW deformations, in positive characteristic particularly, more conceptually in terms of Alexander-Whitney and Eilenberg-Zilber maps twisted by group actions.

We begin in Section 2 by recalling a construction of twisted product resolutions for skew group algebras. In Section 3, we define the group-twisted Alexander-Whitney and Eilenberg-Zilber maps informing deformation theory. We prove in Section 4 our main Theorem 4.1 and show that composing with these maps provides a way to embed convenient resolutions into bar resolutions as direct summand subcomplexes, generalizing results from earlier papers. In Section 5, we explain how some previous results in deformation theory follow from our main theorem in a more conceptual way.

Throughout, $k$ will be a field of arbitrary characteristic, and $\otimes=\otimes_{k}$.

Date: April 19, 2020.

2010 Mathematics Subject Classification. 16E40.

Key words: deformation theory, skew group algebras, Hochschild cohomology.

The first author was partially supported by Simons grant 429539. The second author was partially supported by NSF grant DMS-1665286. Corresponding Author: Anne Shepler. 


\section{RESOLUtions FOR SKEW GROUP ALGEBRAS}

Consider a finite group $G$ acting on a $k$-algebra $S$ by automorphisms. The resulting skew group algebra $S \rtimes G$ is the free $S$-module with basis $G$ and multiplication

$$
(s g) \cdot\left(s^{\prime} g^{\prime}\right)=s\left({ }^{g} s^{\prime}\right) g g^{\prime} \quad \text { for all } \quad s, s^{\prime} \in S \text { and } g, g^{\prime} \in G
$$

where ${ }^{g} s^{\prime}$ denotes the action of $g$ on $s^{\prime}$. We recall a construction from [21, 23].

The twisted product resolution. Consider projective resolutions

(i) $\quad C_{.}: \quad \ldots \rightarrow C_{2} \rightarrow C_{1} \rightarrow C_{0} \rightarrow 0$ of $k G$ as a $k G$-bimodule, and

(ii) $\quad D_{.}: \quad \ldots \rightarrow D_{2} \rightarrow D_{1} \rightarrow D_{0} \rightarrow 0$ of $S$ as an $S$-bimodule.

We take $C$. to be $G$-graded, with group action compatible with the grading, that is,

$$
g_{1}\left(\left(C_{i}\right)_{g_{2}}\right) g_{3}=\left(C_{i}\right)_{g_{1} g_{2} g_{3}} \quad \text { for all } g_{1}, g_{2}, g_{3} \in G \text { and all degrees } i,
$$

and choose a resolution $D$. upon which $G$ acts: Each $D_{i}$ should be a left $k G$-module with $g \cdot(s \cdot d)={ }^{g} s \cdot(g \cdot d)$ for $g$ in $G, s$ in $S$, and $d$ in $D$ and with differentials $k G$-module homomorphisms. Then $D$. is said to be compatible with the twisting map given by the group action (see [23, Definition 2.17]). For example, these conditions all hold when $C$. is the bar resolution or reduced bar resolution of $k G$ and $D$. is the Koszul resolution of a Koszul algebra $S$ with action of $G$ by automorphisms (see [23, Prop 2.20(ii)]).

We combine the above resolutions of $k G$ and $S$ to construct a resolution of $S \rtimes G$ : The twisted product resolution $C \otimes \otimes^{G} D$ of the algebra $S \rtimes G$ is given as a complex of vector spaces by the total complex of the double complex $C . \otimes D$. :

$$
\left(C \otimes \otimes^{G} D\right)_{n}=\bigoplus_{i+j=n} C_{i} \otimes D_{j}
$$

We imbue $C \otimes^{G} D$ with an $(S \rtimes G)$-bimodule structure on its components by defining an action of $S$ on the left and an action of $k G$ on the right given by

$$
s \cdot(c \otimes d) \cdot h=c h \otimes{ }^{(g h)^{-1}} s \cdot h^{-1} d \quad \text { for } g, h \in G, c \in C_{g}, d \in D, s \in S .
$$

Then the complex $C \otimes{ }^{G} D$, augmented by $S \rtimes G$, is indeed an exact sequence of $(S \rtimes G)$ bimodules (see [23] or [20, §4]). It is a projective resolution of $S \rtimes G$ when each $(C \otimes G D)_{n}$ is projective as a $(S \rtimes G)$-bimodule. This is the case, for example, when $D$ is a Koszul resolution of a Koszul algebra and $C$ is the bar resolution of $k G$. In this case, each $D_{j}$ is a free $S$-module and each $C_{i}$ is a free $k G$-module, and it can be shown directly that $C_{i} \otimes D_{j}$ is a free $(S \rtimes G)$ )-bimodule (with basis given by tensoring the basis elements of $C_{i}$ with those of $D_{j}$ ).

Reduced bar resolutions. We now consider a special case of the twisted product resolution. Fix $C=\bar{B}_{G}$ and $D=\bar{B}_{S}$, the reduced bar resolutions for $G$ and $S$, respectively:

$$
\left(\bar{B}_{G}\right)_{n}=k G \otimes \overline{k G}^{\otimes n} \otimes k G \quad \text { and } \quad\left(\bar{B}_{S}\right)_{n}=S \otimes \bar{S}^{\otimes n} \otimes S
$$

where $\overline{k G}=k G /\left(k \cdot 1_{G}\right)$ as a vector space, and similarly $\bar{S}=S /\left(k \cdot 1_{S}\right)$. We fix some choice of section $\bar{S} \hookrightarrow S$ and choose the embedding $G-\{1\} \hookrightarrow G$ to define a section 
$\overline{k G} \hookrightarrow k G$. There is a twisted product resolution (see [23, Proposition 2.20(ii) and Corollary 3.12]) of $S \rtimes G$ formed by these reduced bar complexes:

$$
C \otimes^{G} D=\bar{B}_{G} \otimes^{G} \bar{B}_{S} .
$$

It is given as a vector space in degree $n$ by

$$
\left(\bar{B}_{G} \otimes{ }^{G} \bar{B}_{S}\right)_{n}=\bigoplus_{\ell=0}^{n} k G \otimes(\overline{k G})^{\otimes(n-\ell)} \otimes k G \otimes S \otimes \bar{S}^{\otimes \ell} \otimes S .
$$

We compare this twisted product resolution of $S \rtimes G$ with its reduced bar resolution $\bar{B}_{S \rtimes G}$. Here, $\overline{(S \rtimes G)}=(S \rtimes G) /\left(k \cdot 1_{S \rtimes G}\right)$ with $1_{S \rtimes G}=1_{S} \cdot 1_{G}$ and

$$
\left(\bar{B}_{S \rtimes G}\right)_{n}=(S \rtimes G) \otimes \overline{(S \rtimes G)}^{\otimes n} \otimes(S \rtimes G) .
$$

\section{Group-twisted Alexander-Whitney and Eilenberg-Zilber maps}

We now define analogs of the Alexander-Whitney and Eilenberg-Zilber maps in the environment of group actions. (Compare with [12, 13] for the case of tensor products of algebras and [11] for twisted tensor products defined by bicharacters on grading groups.)

Recall that the traditional Alexander-Whitney and Eilenberg-Zilber maps convert between the reduced bar resolution of a tensor product and the tensor product of the individual reduced bar resolutions:

$$
\begin{aligned}
\mathrm{AW}_{n}: & \left(\bar{B}_{S \otimes k G}\right)_{n} \\
\mathrm{EZ}_{n}: \quad\left(\bar{B}_{S} \otimes \bar{B}_{G}\right)_{n} & \longrightarrow\left(\bar{B}_{G} \otimes \bar{B}_{S \otimes k G}\right)_{n},
\end{aligned}
$$

We define analogs of these maps for the skew group algebra $S \rtimes G$. This algebra is $S \otimes k G$ as a vector space but with multiplication given by a twisting map $k G \otimes S \rightarrow S \otimes k G$. Consequently, we switch the order of $k G$ and $S$ when taking the tensor products of the individual bar resolutions. We define maps, for each $n$,

$$
\begin{aligned}
\mathrm{AW}_{n}^{G}: & \left(\bar{B}_{S \rtimes G}\right)_{n} \\
\mathrm{EZ}_{n}^{G}:\left(\bar{B}_{G} \otimes^{G} \bar{B}_{S}\right)_{n} & \longrightarrow\left(\bar{B}_{G} \otimes^{G} \bar{B}_{S}\right)_{n},
\end{aligned}
$$

For ease of notation, we write elements of $\overline{k G}$ and $\bar{S}$ just as if they were in $k G$ and $S$, respectively, invoking our choices of section maps, and no confusion should arise.

Group-twisted Alexander-Whitney map. Define the first map by

$$
\begin{aligned}
\mathrm{AW}_{n}^{G}\left(1 \otimes s_{1} g_{1}\right. & \left.\otimes \cdots \otimes s_{n} g_{n} \otimes 1\right) \\
= & \sum_{\ell=0}^{n}(-1)^{\ell(n-\ell)}\left(g_{1} \cdots g_{\ell}\right) \otimes g_{\ell+1} \otimes \cdots \otimes g_{n} \otimes 1 \otimes \\
& \quad 1 \otimes\left(g_{1} \cdots g_{n}\right)^{-1} s_{1} \otimes \cdots \otimes\left(g_{\ell} \cdots g_{n}\right)^{-1} s_{\ell} \otimes\left(g_{\ell+1} \cdots g_{n}\right)^{-1}\left(s_{\ell+1} \cdots s_{n}\right)
\end{aligned}
$$

for $g_{1}, \ldots, g_{n}$ in $G$ and $s_{1}, \ldots, s_{n}$ in $S$. Here, any tensor component in $k \cdot 1_{G}$ (respectively, $k \cdot 1_{S}$ or $k \cdot 1_{S \rtimes G}$ ) is understood to be zero as an element of $\overline{k G}$ (respectively, $\bar{S}$ or $\overline{S \rtimes G}$ ). For each $n$, this defines the group-twisted Alexander-Whitney map $\mathrm{AW}_{n}^{G}$ as an $(S \rtimes G)$ bimodule homomorphism, since it is defined on a free basis. 
Group-twisted Eilenberg-Zilber map. We shuffle the elements of $G$ with the elements of $S$ to define the second map. Let $\mathfrak{S}_{n-\ell, \ell}$ be the set of $(n-\ell, \ell)$-shuffles in the symmetric group $\mathfrak{S}_{n}$, i.e., permutations $\sigma$ of $1, \ldots, n$ with

$$
\sigma(1)<\sigma(2)<\cdots<\sigma(n-\ell) \quad \text { and } \quad \sigma(n-\ell+1)<\sigma(n-\ell+2)<\cdots<\sigma(n) .
$$

We also count the number of inversions of a permutation $\sigma$ in $\mathfrak{S}_{n}$ : Set

$$
|\sigma|=\mid\{(i, j) \mid 1 \leq i<j \leq n \text { and } \sigma(i)>\sigma(j)\} \mid .
$$

For each shuffle $\sigma^{-1} \in \mathfrak{S}_{n-\ell, \ell}$ and tuple $\left(g_{1}, \ldots, g_{n-\ell}, s_{1}, \ldots, s_{\ell}\right)=\left(x_{1}, \ldots, x_{n}\right)$, define

$$
F_{\sigma}\left(x_{1} \otimes \cdots \otimes x_{n}\right)=h_{\sigma(1)}\left(x_{\sigma(1)}\right) \otimes \cdots \otimes h_{\sigma(n)}\left(x_{\sigma(n)}\right)
$$

where $h_{i}=1_{G}$ for $1 \leq i \leq n-\ell\left(\right.$ so ${ }^{h_{i}} x_{i}=x_{i}$ for $\left.x_{i} \in G\right)$ and

$$
h_{i}=\prod_{\substack{\sigma^{-1}(i)+1 \leq j \leq n \\ \sigma(j) \leq n-\ell}} g_{\sigma(j)} \text { in } G \quad \text { for } n-\ell+1 \leq i \leq n \quad\left(\text { when } x_{i} \in S\right) .
$$

We define the group-twisted Eilenberg-Zilber map by

$$
\begin{aligned}
\mathrm{EZ}_{n}^{G}\left(1 \otimes g_{1} \otimes \cdots \otimes g_{n-\ell} \otimes 1 \otimes 1 \otimes s_{1} \otimes \cdots \otimes s_{\ell} \otimes 1\right) \\
\quad=\sum_{\sigma^{-1} \in \mathfrak{S}_{n-\ell, \ell}}(-1)^{|\sigma|} \otimes F_{\sigma}\left(g_{1} \otimes \cdots \otimes g_{n-\ell} \otimes s_{1} \otimes \cdots \otimes s_{\ell}\right) \otimes 1
\end{aligned}
$$

for all $g_{1}, \ldots, g_{n-\ell}$ in $G-\{1\}$ and $s_{1}, \ldots, s_{\ell}$ in $\bar{S}$. For each $n$, this defines $\mathrm{EZ}_{n}^{G}$ as an $(S \rtimes G)$-bimodule homomorphism, since it is defined on a free basis.

Group-twisted maps are chain maps. These group-twisted maps convert between two resolutions of the algebra $S \rtimes G$ : the reduced bar resolution $\bar{B}_{S \rtimes G}$ on one hand and the twisted product resolution $\bar{B}_{G} \otimes{ }^{G} \bar{B}_{S}$ on the other hand.

Lemma 3.1. The group-twisted maps $\mathrm{AW}_{n}^{G}$ and $\mathrm{EZ}_{n}^{G}$ are chain maps:

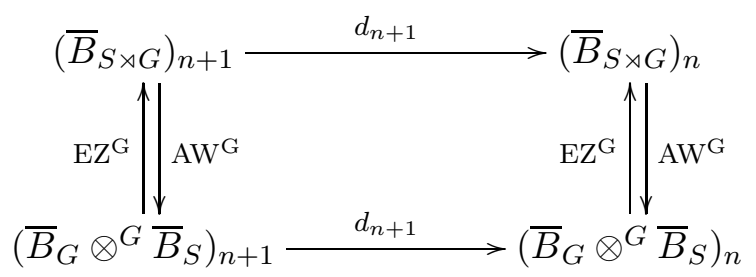

Proof. A calculation shows that $\mathrm{AW}^{G}$ is a chain map just as in the case of the traditional Alexander-Whitney map for the tensor product of algebras (see, e.g., [16, (X.7.2)]). For 
example, in degree 2 ,

$$
\begin{aligned}
d_{2} \mathrm{AW}_{2}^{G}\left(1 \otimes s_{1} g_{1} \otimes s_{2} g_{2} \otimes 1\right) & \\
=d_{2}( & \left(1 \otimes g_{1} \otimes g_{2} \otimes 1\right) \otimes\left(1 \otimes g_{1}^{-1} g_{2}^{-1} s_{1}^{g_{2}^{-1}} s_{2}\right)-\left(g_{1} \otimes g_{2} \otimes 1\right) \otimes\left(1 \otimes g_{1}^{-1} g_{2}^{-1} s_{1} \otimes g_{2}^{-1} s_{2}\right) \\
& \left.\quad+\left(g_{1} g_{2} \otimes 1\right) \otimes\left(1 \otimes g_{1}^{-1} g_{2}^{-1} s_{1} \otimes g_{2}^{-1} s_{2} \otimes 1\right)\right) \\
=\left(g_{1} \otimes\right. & \left.g_{2} \otimes 1\right) \otimes\left(1 \otimes g_{1}^{-1} g_{2}^{-1} s_{1} g_{2}^{-1} s_{2}\right)-\left(1 \otimes g_{1} g_{2} \otimes 1\right) \otimes\left(1 \otimes g_{1}^{-1} g_{2}^{-1} s_{1}^{g_{2}^{-1}} s_{2}\right) \\
& +\left(1 \otimes g_{1} \otimes g_{2}\right) \otimes\left(1 \otimes{ }_{1}^{-1} g_{2}^{-1} s_{1} g_{2}^{-1} s_{2}\right)-\left(g_{1} g_{2} \otimes 1\right) \otimes\left(1 \otimes{ }_{1}^{-1} g_{2}^{-1} s_{1} \otimes g_{2}^{-1} s_{2}\right) \\
& +\left(g_{1} \otimes g_{2}\right) \otimes\left(1 \otimes g_{1}^{-1} g_{2}^{-1} s_{1} \otimes^{g_{2}^{-1}} s_{2}\right)+\left(g_{1} \otimes g_{2} \otimes 1\right) \otimes\left({ }^{-1} g_{2}^{-1} s_{1} \otimes g_{2}^{-1} s_{2}\right) \\
& -\left(g_{1} \otimes g_{2} \otimes 1\right) \otimes\left(1 \otimes g_{1}^{-1} g_{2}^{-1} s_{1} g_{2}^{-1} s_{2}\right)+\left(g_{1} g_{2} \otimes 1\right) \otimes\left(g_{1}^{-1} g_{2}^{-1} s_{1} \otimes g_{2}^{-1} s_{2} \otimes 1\right) \\
& -\left(g_{1} g_{2} \otimes 1\right) \otimes\left(1 \otimes g_{1}^{-1} g_{2}^{-1} s_{1} g_{2}^{-1} s_{2} \otimes 1\right)+\left(g_{1} g_{2} \otimes 1\right) \otimes\left(1 \otimes g_{1}^{-1} g_{2}^{-1} s_{1} \otimes g_{2}^{-1} s_{2}\right),
\end{aligned}
$$

whereas

$$
\begin{aligned}
& \mathrm{AW}_{1}^{G} d_{2}\left(1 \otimes s_{1} g_{1} \otimes s_{2} g_{2} \otimes 1\right) \\
& =\mathrm{AW}_{1}\left(s_{1} g_{1} \otimes s_{2} g_{2} \otimes 1-1 \otimes s_{1}{ }^{g_{1}} s_{2} g_{1} g_{2} \otimes 1+1 \otimes s_{1} g_{1} \otimes s_{2} g_{2}\right) \\
& =s_{1} g_{1}\left(\left(1 \otimes g_{2} \otimes 1\right) \otimes\left(1 \otimes g_{2}^{-1} s_{2}\right)+\left(g_{2} \otimes 1\right) \otimes\left(1 \otimes g^{g^{-1}} s_{2} \otimes 1\right)\right) \\
& -\left(1 \otimes g_{1} g_{2} \otimes 1\right) \otimes\left(1 \otimes g_{1}^{-1} g_{2}^{-1} s_{1} g_{2}^{-1} s_{2}\right)-\left(g_{1} g_{2} \otimes 1\right) \otimes\left(1 \otimes g_{1}^{-1} g_{2}^{-1} s_{1}{ }^{-1} s_{2} \otimes 1\right) \\
& +\left(\left(1 \otimes g_{1} \otimes 1\right) \otimes\left(1 \otimes g_{1}^{-1} s_{1}\right)+\left(g_{1} \otimes 1\right) \otimes\left(1 \otimes g^{g_{1}^{-1}} s_{1} \otimes 1\right)\right) s_{2} g_{2} \\
& =\left(g_{1} \otimes g_{2} \otimes 1\right) \otimes\left({ }^{g_{1}^{-1}} g_{2}^{-1} s_{1} \otimes{ }^{g_{2}^{-1}} s_{2}\right)+\left(g_{1} g_{2} \otimes 1\right) \otimes\left({ }^{g_{1}^{-1}} g_{2}^{-1} s_{1} \otimes{ }^{g_{2}^{-1}} s_{2} \otimes 1\right) \\
& -\left(1 \otimes g_{1} g_{2} \otimes 1\right) \otimes\left(1 \otimes g_{1}^{-1} g_{2}^{-1} s_{1} g_{2}^{-1} s_{2}\right)-\left(g_{1} g_{2} \otimes 1\right) \otimes\left(1 \otimes g_{1}^{-1} g_{2}^{-1} s_{1} g^{-1} s_{2} \otimes 1\right) \\
& +\left(1 \otimes g_{1} \otimes g_{2}\right) \otimes\left(1 \otimes g_{1}^{-1} g_{2}^{-1} s_{1}^{g_{2}^{-1}} s_{2}\right)+\left(g_{1} \otimes g_{2}\right) \otimes\left(1 \otimes g_{1}^{-1} g_{2}^{-1} s_{1} \otimes g_{2}^{-1} s_{2}\right),
\end{aligned}
$$

and we see that these two expressions are equal after some cancellations. Similarly, to show $\mathrm{EZ}^{G}$ is a chain map, we follow the proof that the classical Eilenberg-Zilber map EZ is a chain map but include the group action. (See, e.g., [16, (VIII.8.9)] and [13.)

Next we see that the maps $\mathrm{AW}^{G}, \mathrm{EZ}^{G}$ provide a splitting of the reduced bar resolution of $S \rtimes G$, with a copy of the resolution $\bar{B}_{G} \otimes{ }^{G} \bar{B}_{S}$ as a direct summand.

Lemma 3.2. $\mathrm{AW}^{G} \mathrm{EZ}^{G}$ is the identity map in each degree.

Proof. If the action of $G$ is trivial, we have the standard Alexander-Whitney and Eilenberg-Zilber maps, and $\mathrm{AW}^{1} \mathrm{EZ}^{1}$ is known to be the identity map 13, Remark 3.2]. One can verify that $\mathrm{AW}^{G} \mathrm{EZ}^{G}$ is the identity map for nontrivial group actions as well, via a straightforward but tedious calculation, keeping careful track of group actions. For example, in degree 2, for all $g$ in $G$ and $s$ in $S$,

$$
\begin{aligned}
\mathrm{AW}_{2}^{G} \mathrm{EZ}_{2}^{G}((1 \otimes g \otimes 1) \otimes(1 \otimes s \otimes 1)) & =\mathrm{AW}_{2}^{G}\left(1 \otimes g \otimes s \otimes 1-1 \otimes{ }^{g} s \otimes g \otimes 1\right) \\
& =(1 \otimes g \otimes 1) \otimes(1 \otimes s \otimes 1) .
\end{aligned}
$$

Note that many terms in the image of $\mathrm{AW}_{2}^{G}$ are 0 since we are working with the reduced (instead of unreduced) bar resolution. 


\section{Conversion Between Resolutions}

In this section, we show that the group-twisted Alexander-Whitney and EilenbergZilber maps provide a way to convert between the reduced bar resolution for a skew group algebra $S \rtimes G$ and a choice of twisted product resolution for $S \rtimes G$, which generally can be much smaller. Compare to [22, Lemma 4.4] for Koszul algebras; our result here is stated for more general algebras $S$, and we provide a proof based on the explicit chain maps $\mathrm{AW}^{G}$ and $\mathrm{EZ}^{G}$ defined above. We will see in the next section that the maps $\mathrm{AW}^{G}$ and $\mathrm{EZ}^{G}$ have deformation-theoretic consequences.

Again, consider compatible projective resolutions

(i) $\quad C_{.}: \quad \ldots \rightarrow C_{2} \rightarrow C_{1} \rightarrow C_{0} \rightarrow 0$ of $k G$ as a $k G$-bimodule, and

(ii) $\quad D_{.}: \quad \ldots \rightarrow D_{2} \rightarrow D_{1} \rightarrow D_{0} \rightarrow 0$ of $S$ as an $S$-bimodule

as in Section 2 and their twisted product resolution $C \otimes^{G} D$. We combine the grouptwisted Alexander-Whitney and Eilenberg-Zilber maps with chain maps converting between $C, D$ and the reduced bar resolutions $\bar{B}_{G}, \bar{B}_{S}$, respectively, to construct a chain map splitting

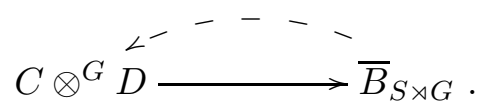

We take the setting when chain maps

$$
\iota_{G}: C \rightarrow \bar{B}_{G}, \quad \pi_{G}: \bar{B}_{G} \rightarrow C, \quad \iota_{S}: D \rightarrow \bar{B}_{S}, \quad \pi_{S}: \bar{B}_{S} \rightarrow D
$$

are given for which $\iota_{S}, \pi_{S}$ are $k G$-module homomorphisms and $\pi_{G} \iota_{G}$ and $\pi_{S} \iota_{S}$ are identity maps in each degree:
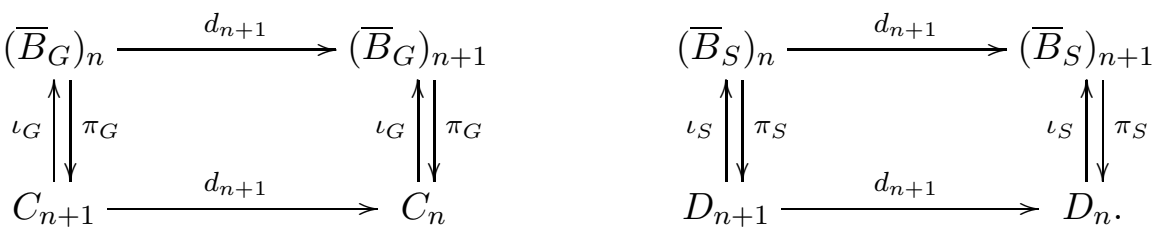

For example, if $S=S(V)$ is a symmetric algebra on a finite dimensional vector space $V$ with an action of $G$ by automorphisms, and $D$ is the Koszul resolution of $S$, maps $\iota_{S}, \pi_{S}$ are constructed explicitly in [19, (2.6) and (4.2)], and the hypotheses of the next theorem hold.

Theorem 4.1. Let $G$ be a finite group acting on a $k$-algebra $S$ by automorphisms. Let $\bar{B}_{S \rtimes G}$ be the reduced bar resolution for $S \rtimes G$. Let $C \otimes^{G} D$ be a twisted product resolution for $S \rtimes G$ obtained by twisting together a resolution $C$. of $k G$ and a resolution $D$. of $S$ as above. There exist chain maps

$$
\iota:\left(C \otimes^{G} D\right) . \rightarrow\left(\bar{B}_{S \rtimes G}\right) . \quad \text { and } \quad \pi:\left(\bar{B}_{S \rtimes G}\right) . \rightarrow\left(C \otimes^{G} D\right) .
$$

for which $\pi_{n} \iota_{n}:\left(C \otimes^{G} D\right)_{n} \rightarrow\left(C \otimes^{G} D\right)_{n}$ is the identity map for all $n$. If $S$ is a graded algebra and $G$ consists of elements of degree 0 , then the chain maps $\iota, \pi$ are graded maps provided $\iota_{S}$ and $\pi_{S}$ are graded maps. 
Proof. Define compositions

$$
\iota=\mathrm{EZ}^{G}\left(\iota_{G} \otimes \iota_{S}\right) \quad \text { and } \quad \pi=\left(\pi_{G} \otimes \pi_{S}\right) \mathrm{AW}^{G},
$$

which can be visualized via a diagram:

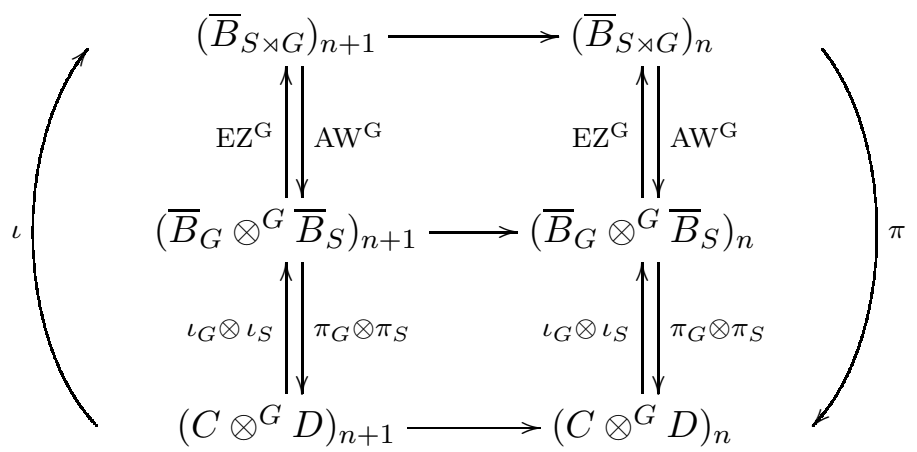

By Lemmas 3.1 and 3.2 and the hypotheses on $\iota_{G}, \iota_{S}, \pi_{G}, \pi_{S}$, the composition $\pi \iota$ is 1 .

\section{Applications to Deformation theory}

We now consider some applications of Theorem 4.1. We used Hochschild cohomology to analyze PBW deformations of $S \rtimes G$ in [21] and [22], particularly in the case that $S=S(V)$ is a symmetric algebra. Our Theorem 4.1 generalizes both [21, Lemma 7.3] and [22, Lemma 4.4], which provide an explicit connection between Hochschild cocycles and PBW deformations. We explain here how Theorem 4.1 gives a unified proof of this connection using the group-twisted Alexander-Whitney and Eilenberg-Zilber maps. This approach is more elegant than the somewhat ad hoc proofs given in [21, 22]. It also more easily applies to other settings. We emphasize that we did not assume $S$ is Koszul in Theorem 4.1 in the last section, although $S$ will be a Koszul algebra here.

Let $V$ be a finite dimensional $k G$-module and $S=S(V)$, the symmetric algebra on the underlying vector space $V$. Consider some linear parameter functions

$$
\kappa: V \otimes V \rightarrow k G \quad \text { and } \quad \lambda: k G \otimes V \rightarrow k G
$$

with $\kappa$ alternating. We view $\kappa$ as a function on the exterior power $\bigwedge^{2} V$.

Let $\mathcal{H}_{\lambda, \kappa}$ be the associative $k$-algebra generated by a basis of $V$ and the group algebra $k G$ with relations given by those of $k G$ together with

$$
v w-w v=\kappa(v \wedge w) \quad \text { and } \quad g v-{ }^{g} v g=\lambda(g \otimes v) \quad \text { for } v, w \in V, g \in G .
$$

Then $\mathcal{H}_{\lambda, \kappa}$ is a filtered algebra with $\operatorname{deg}(v)=1$ and $\operatorname{deg}(g)=0$ for all $v$ in $V$ and $g$ in $G$. We call $\mathcal{H}_{\lambda, \kappa}$ a $P B W$ deformation of $S(V) \rtimes G$ if gr $\mathcal{H}_{\lambda, \kappa} \cong S(V) \rtimes G$, that is, the associated graded algebra of $\mathcal{H}_{\lambda, \kappa}$ is isomorphic to the skew group algebra.

Over fields $k$ of characteristic 0 , the PBW deformations $\mathcal{H}_{\lambda, \kappa}$ with $\kappa \equiv 0$ include the affine graded Hecke algebras defined by Lusztig [14, 15]; those with $\lambda \equiv 0$ include the symplectic reflection algebras and Drinfeld Hecke algebras (see [8, 10, 18]). Note that over fields of characteristic 0 (or more generally coprime to the group order $|G|$ ), every PBW algebra of the form $\mathcal{H}_{\lambda, 0}$ is isomorphic to one of the form $\mathcal{H}_{0, \kappa}$, but this fact does not hold in positive characteristic (see [21, Theorem 4.1, Example 5.1]). 
The PBW property imposes conditions on $\kappa, \lambda$, as given in the following theorem, which is [21, Theorem 3.1]. Observe that when $\lambda$ is zero, all but conditions (2) and (4) in the theorem are trivial.

Theorem 5.1. Let $k$ be a field of arbitrary characteristic. The algebra $\mathcal{H}_{\lambda, \kappa}$ is a PBW deformation of $S(V) \rtimes G$ if and only if the following conditions hold for all $g, h$ in $G$ and $u, v, w$ in $V$.

(1) $\lambda(g h, v)=\lambda\left(g,{ }^{h} v\right) h+g \lambda(h, v)$ in $k G$.

(2) $\quad \kappa\left({ }^{g} u,{ }^{g} v\right) g-g \kappa(u, v)=\lambda(\lambda(g, v), u)-\lambda(\lambda(g, u), v)$ in $k G$.

(3) $\quad \lambda_{h}(g, v)\left({ }^{h} u-{ }^{g} u\right)=\lambda_{h}(g, u)\left({ }^{h} v-{ }^{g} v\right)$ in $V$.

(4) $\kappa_{g}(u, v)\left({ }^{g} w-w\right)+\kappa_{g}(v, w)\left({ }^{g} u-u\right)+\kappa_{g}(w, u)\left({ }^{g} v-v\right)=0$ in $V$.

(5) $\lambda(\kappa(u, v), w)+\lambda(\kappa(v, w), u)+\lambda(\kappa(w, u), v)=0$ in $k G$.

The five conditions in Theorem 5.1 may not seem intuitive at first glance, and the proof given in [21] indeed uses the Diamond Lemma (see [5] and [4]). Hochschild cohomology gives a natural interpretation for the five conditions in terms of Gerstenhaber brackets and cocycles, as explained in [21, Section 8]. There, we considered a chain map from a twisted product resolution $X$. to the bar resolution,

$$
\phi_{.}: X_{\bullet} \longrightarrow B_{S \rtimes G},
$$

where $X_{.}=C \otimes{ }^{G} D$ is defined as in (2.1) for $C$ the bar resolution of $k G$ and $D$ the Koszul resolution of $S$. We used this chain map to translate the five conditions of Theorem 5.1 into provisions more natural in the architecture of cohomology. But the chain map $\phi$. was constructed there in an improvised and utilitarian way. We now explain how to use Theorem 4.1 instead to give a direct proof of [21, Theorem 8.3]:

Theorem 5.2. Let $k$ be a field of arbitrary characteristic. The algebra $\mathcal{H}_{\lambda, \kappa}$ exhibits the $P B W$ property if and only if

- $d^{*}(\lambda)=0$,

- $[\lambda, \lambda]=2 d^{*}(\kappa)$, and

- $[\lambda, \kappa]=0$

as Hochschild cochains, where $\lambda$ and $\kappa$ are identified with cochains on the resolution $X$. .

Proof. Let $\operatorname{Kosz}_{S(V)}$ be the Koszul resolution of the polynomial ring $S(V)$ and set

$$
S=S(V), \quad D=\operatorname{Kosz}_{S(V)}, \quad C=\bar{B}_{k G}, \quad \text { and } X=C \otimes^{G} D .
$$

We take the standard embedding $\iota_{S}=\operatorname{Kosz}_{S} \hookrightarrow \bar{B}_{S}$ and set $\iota_{C}=1$, the identity map on $\bar{B}_{k G}$. Then Theorem 4.1 implies existence of maps

$$
\iota:\left(C \otimes^{G} D\right) . \rightarrow\left(\bar{B}_{S \rtimes G}\right) . \quad \text { and } \quad \pi:\left(\bar{B}_{S \rtimes G}\right) . \rightarrow\left(C \otimes^{G} D\right) .
$$

for which $\pi \iota$ is the identity map in each degree. The map $\phi$. constructed in [21, Lemma 7.3] is essentially our map $\iota$ : There, $C$ was the unreduced bar resolution of $k G$, whereas here we use the reduced bar resolution instead so that $\mathrm{AW}^{G} \mathrm{EZ}^{G}=1$ in general, avoiding the need for [21, Lemma 7.3]. 
We identify the parameter $\lambda$ of the algebra $H_{\lambda, \kappa}$ with a cochain in

$$
\operatorname{Hom}_{(S \rtimes G)^{e}}\left(X_{1,1}, S \rtimes G\right),
$$

in the following way: Since $\lambda$ factors through the quotient $k G \otimes V \rightarrow \overline{k G} \otimes V$, we may view $\lambda$ as a function $X_{1,1} \rightarrow k G$; composing with the chain map $\pi$ from Theorem 4.1 gives a cochain $\mu_{1}=\pi \otimes \lambda$ in $\operatorname{Hom}_{(S \rtimes G)^{e}}\left(\bar{B}_{S \rtimes G}, S \rtimes G\right)$ for which

$$
\mu_{1}(1 \otimes g \otimes v \otimes 1)-\mu_{1}\left(1 \otimes{ }^{g} v \otimes g \otimes 1\right)=\lambda(g \otimes v) \quad \text { for } g \in G, v \in V .
$$

Here, one recycles part of the calculation from the proof of Lemma 3.2. Note that $(S \rtimes G)^{e}$ denotes the enveloping algebra $(S \rtimes G) \otimes(S \rtimes G)^{\mathrm{op}}$.

Similarly, we identify $\kappa$ with a cochain $\mu_{2}$ in $\operatorname{Hom}_{(S \rtimes G)^{e}}\left(X_{0,2}, S \rtimes G\right)$ by composing again with $\pi$; specifically, $\mu_{2}$ in $\operatorname{Hom}_{(S \rtimes G)^{e}}\left(\bar{B}_{S \rtimes G}, S \rtimes G\right)$ is the cochain for which

$$
\kappa(v \wedge w)=\mu_{2}(1 \otimes v \otimes w \otimes 1)-\mu_{2}(1 \otimes w \otimes v \otimes 1) \quad \text { for } v, w \in V .
$$

Deformation theory gives Hochschild conditions necessary for a cochain on the bar resolution of an algebra to define a deformation of that algebra (see [9]). We apply these conditions specifically to the cochains $\mu_{1}, \mu_{2}$ and then translate via $\iota$ to conditions on $\lambda, \kappa$. We find that Conditions (1) and (3) of Theorem 5.1 hold exactly when $\lambda$ is a cocycle as evaluated on $X_{2,1}$ and on $X_{1,2}$, i.e., $d^{*}(\lambda)=0$. Conditions (2) and (4) hold exactly when the Gerstenhaber bracket $[\lambda, \lambda]$ coincides with $2 d^{*}(\kappa)$ as evaluated on $X_{1,2}$ and on $X_{0,3}$. Condition (5) holds exactly when the Gerstenhaber bracket $[\lambda, \kappa]$ vanishes. Details are in the proof of [21, Theorem 8.3].

Remark 5.3. While full details of the proof of Theorem [5.2 are in [21], we emphasize that our contribution here is to show that the needed chain maps arise automatically from group-twisted Alexander-Whitney and Eilenberg-Zilber maps. In this way, these maps provide a general method for converting from Diamond Lemma conditions to homological conditions, giving a more unified and less ad hoc approach toward studying deformations. We expect that this approach will lend itself to interesting generalizations.

Remark 5.4. Similarly, in 22, we consider the case when $S$ is a quadratic algebra, and especially the case when $S$ is Koszul. Again, we used an explicit chain map established with improvised methods (see [22, Lemmas 4.4 and 4.6]) to convert between resolutions and study explicit conditions giving PBW deformations of $S \rtimes G$. Indeed, the proof of [22, Theorem 2.5] relies on explicit information provided by chain maps. Here again, chain maps may be taken from Theorem 4.1 instead to give a more conceptual view of the deformation-theoretic results in [22].

\section{REFERENCES}

[1] M. Balagovic and H. Chen, "Representations of rational Cherednik algebras in positive characteristic," J. Pure Appl. Algebra 217 (2013), no. 4, 716-740.

[2] M. Balagovic and H. Chen, "Category $\mathcal{O}$ for rational Cherednik algebras $H_{t, c}\left(\mathrm{GL}_{2}\left(\mathbb{F}_{p}\right), \mathfrak{h}\right)$ in characteristic $p, "$ J. Pure Appl. Algebra 217 (2013), no. 9, 1683-1699.

[3] G. Bellamy and M. Martino, "On the smoothness of centres of rational Cherednik algebras in positive characteristic." Glasgow Math. Journal 55 (2013), no. A, 27-54.

[4] G. Bergman, "The diamond lemma for ring theory," Adv. Math. 29 (1978) no. 2, 178-218. 
[5] L. A. Bokut', "Embeddings into simple associative algebras," (Russian) Algebra i Logika 15 (1976), no. 2, 117-142, 245. English translation in Algebra and Logic, 73-90.

[6] K. A. Brown and K. Changtong, "Symplectic reflection algebras in positive characteristic," Proc. Edinb. Math. Soc. (2) 53 (2010), no. 1, 61-81.

[7] V. G. Drinfeld, "Degenerate affine Hecke algebras and Yangians," Funct. Anal. Appl. 20 (1986), $58-60$.

[8] P. Etingof and V. Ginzburg, "Symplectic reflection algebras, Calogero-Moser space, and deformed Harish-Chandra homomorphism," Invent. Math. 147 (2002), no. 2, 243-348.

[9] M. Gerstenhaber and S. Schack, "Algebraic cohomology and deformation theory," Deformation theory of algebras and structures and applications (Il Ciocco, 1986), NATO Adv. Sci. Inst. Ser. C Math. Phys. Sci., 247, Kluwer Acad. Publ., Dordrecht (1988), 11-264.

[10] S. Griffeth, "Towards a combinatorial representation theory for the rational Cherednik algebra of type $G(r, p, n)$," Proceedings of the Edinburgh Mathematical Society (Series 2) 53 (2010), 419-445, see arxiv:0612733.

[11] L. Grimley, V. C. Nguyen, and S. Witherspoon, "Gerstenhaber brackets on Hochschild cohomology of twisted tensor products," J. Noncommutative Geometry 11 (2017), no. 4, 1351-1379.

[12] J. A. Guccione and J. J. Guccione, "Hochschild homology of twisted tensor products," K-Theory 18 (1999), 363-400.

[13] J. Le and G. Zhou, "On the Hochschild cohomology ring of tensor products of algebras," J. Pure Appl. Algebra 218 (2014), 1463-1477.

[14] G. Lusztig, "Cuspidal local systems and graded Hecke algebras. I," Inst. Haute Etudes Sci. Publ. Math. 67 (1988), 145-202.

[15] G. Lusztig, "Affine Hecke algebras and their graded version," J. Amer. Math. Soc. 2 (1989), no. 3, 599-635.

[16] S. Mac Lane, Homology, Springer-Verlag Berlin Heidelberg, 1995, pages: X, 422.

[17] E. Norton, "Symplectic reflection algebras in positive characteristic as Ore extensions," to appear in Communications in Algebra.

[18] A. Ram and A.V. Shepler, "Classification of graded Hecke algebras for complex reflection groups," Comment. Math. Helv. 78 (2003), 308-334.

[19] A.V. Shepler and S. Witherspoon, "Quantum differentiation and chain maps of bimodule complexes," Algebra Number Theory 5-3 (2011), 339-360.

[20] A.V. Shepler and S. Witherspoon, "A Poincaré-Birkhoff-Witt Theorem for quadratic algebras with group actions," Trans. Amer. Math. Soc. 366 (2014), no. 12, 6483-6506.

[21] A.V. Shepler and S. Witherspoon, "PBW deformations of skew group algebras in positive characteristic," Algebras and Representation Theory 18 (2015), no. 1, 257-280.

[22] A.V. Shepler and S. Witherspoon, "Deforming group actions on Koszul algebras," Math. Research Lett. 25 (2018), no. 5, 1613-1644.

[23] A.V. Shepler and S. Witherspoon, "Resolutions for twisted tensor products," Pacific J. Math. 298 (2019), no. 2, 445-469.

Department of Mathematics, University of North Texas, Denton, TX 76203, USA

E-mail address: ashepler@unt.edu

Department of Mathematics, Texas A\&M University, College Station, TX 77843, USA

E-mail address: sjw@math.tamu.edu 